\title{
BMJ
}

\section{Occupational therapy for patients with problems in personal activities of daily living after stroke: systematic review of randomised trials}

\author{
Lynn Legg, CSO research training fellow, ${ }^{1}$ Avril Drummond, principal research fellow in rehabilitation, ${ }^{3}$ \\ Jo Leonardi-Bee, lecturer in medical statistics, ${ }^{2}$ J R F Gladman, professor of medicine of older people, ${ }^{3}$ \\ Susan Corr, reader in occupational science, ${ }^{4}$ Mireille Donkervoort, senior researcher department of \\ rehabilitation medicine, ${ }^{5}$ Judi Edmans, research occupational therapist, ${ }^{3}$ Louise Gilbertson, clinical specialist \\ occupational therapist in stroke, ${ }^{6}$ Lyn Jongbloed, associate professor , ${ }^{7}$ Pip Logan, principal research fellow, ${ }^{3}$ \\ Catherine Sackley, professor of physiotherapy research, ${ }^{8}$ Marion Walker, associate professor and reader in \\ stroke rehabilitation and associate director UK stroke research network, ${ }^{3}$ Peter Langhorne, professor of \\ stroke care ${ }^{1}$
}

\section{${ }^{1}$ Academic Section of Geriatric Medicine, Glasgow Royal Infirmary University NHS Trust, Glasgow G31 2ER \\ ${ }^{2}$ Division of Epidemiology and Public Health, Clinical Sciences Building, City Hospital Campus NHS Trust, Nottingham NG5 1PB \\ ${ }^{3}$ Division of Rehabilitation and Ageing, Medical School, Queen's Medical Centre, Nottingham \\ ${ }^{4}$ Division of Occupational Therapy, School of Health, University of Northampton, Northampton NN2 7AL \\ ${ }^{5}$ Erasmus University Medical Centre, Postbus 2040, 3000 CA, Rotterdam, Netherlands \\ ${ }^{6}$ Occupational Therapy Service, Royal Haslar Hospital, Gosport P012 2AA \\ ${ }^{7}$ Department of Occupational Science and Occupational Therapy, UBC School of Rehabilitation Sciences, Online Programs, University of British Columbia, T325-2211 Wesbrook Mall, Vancouver, BC, Canada V6T 2B5 \\ ${ }^{8}$ Primary Care and General Practice, University of Birmingham, Edgbaston, Birmingham B15 2TT \\ Correspondence to: L Legg step@clinmed.gla.ac.uk}

doi:10.1136/bmj.39343.466863.55

\section{ABSTRACT}

Objective To determine whether occupational therapy focused specifically on personal activities of daily living improves recovery for patients after stroke.

Design Systematic review and meta-analysis.

Data sources The Cochrane stroke group trials register, the Cochrane central register of controlled trials, Medline, Embase, CINAHL, PsycLIT, AMED, Wilson Social Sciences Abstracts, Science Citation Index, Social Science Citation, Arts and Humanities Citation Index, Dissertations Abstracts register, Occupational Therapy Research Index, scanning reference lists, personal communication with authors, and hand searching.

Review methods Trials were included if they evaluated the effect of occupational therapy focused on practice of personal activities of daily living or where performance in such activities was the target of the occupational therapy intervention in a stroke population. Original data were sought from trialists. Two reviewers independently reviewed each trial for methodological quality.

Disagreements were resolved by consensus.

Results Nine randomised controlled trials including 1258 participants met the inclusion criteria. Occupational therapy delivered to patients after stroke and targeted towards personal activities of daily living increased performance scores (standardised mean difference 0.18 , $95 \%$ confidence interval 0.04 to $0.32, \mathrm{P}=0.01$ ) and reduced the risk of poor outcome (death, deterioration or dependency in personal activities of daily living) (odds ratio $0.67,95 \%$ confidence interval 0.51 to 0.87 , $\mathrm{P}=0.003)$. For every 100 people who received occupational therapy focused on personal activities of daily living, 11 (95\% confidence interval 7 to 30 ) would be spared a poor outcome.

Conclusions Occupational therapy focused on improving personal activities of daily living after stroke can improve performance and reduce the risk of deterioration in these abilities. Focused occupational therapy should be available to everyone who has had a stroke.

\section{INTRODUCTION}

Stroke is the second leading cause of death in the world and the leading cause of serious, long term disability in adults; about half of those who survive are dependent on others for assistance with personal activities of daily living six months after the stroke. ${ }^{12}$

Personal activities of daily living are necessary for survival and include "those tasks which all of us undertake every day of our lives in order to maintain our level of care" 3 such as feeding, dressing, toileting, grooming, transferring, and mobilising. ${ }^{4}$

Occupational therapy is an essential element in the rehabilitation of patients after stroke. ${ }^{5}$ It entails "use of purposeful activity or interventions designed to achieve functional outcomes which promote health, prevent injury or disability, and which develop, improve, sustain or restore the highest possible level of independence." 6 Personal activities of daily living is major component of treatment for people who have had a stroke. ${ }^{7}$ Level of dependence in such activities is an important measure of the success of stroke rehabilitation ${ }^{8}$ and a commonly used outcome in stroke trials. ${ }^{4}$

A systematic review of therapy based rehabilitation services delivered to stroke patients living at home within one year of stroke onset ${ }^{9}$ found that those who received rehabilitation based on therapy were more independent in personal activities of daily living and more likely to maintain that ability during the study period. This review, however, covered a heterogeneous group of interventions (physiotherapy, occupational therapy, or multidisciplinary staff working with patients primarily to improve task orientated behaviour) and concluded that the "different groups of interventions might differ in their effects." 
Studies possibly fulfilling inclusion criteria ( $n=14$ 593)

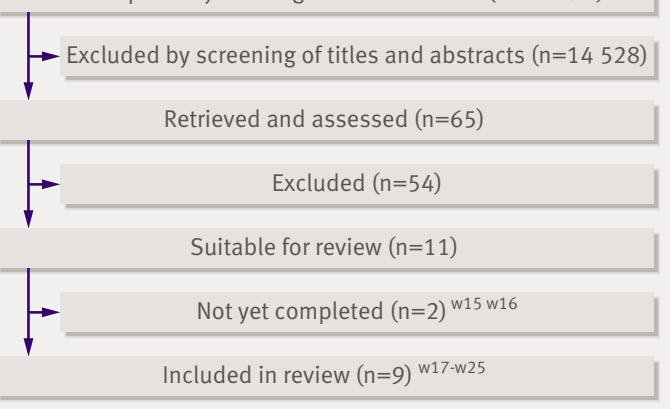

Fig $1 \mid$ Results of literature search and selection of randomised controlled trials for meta-analysis

A subsequent analysis of data from individual patients from eight stroke trials focused on the effect of community occupational therapy on instrumental activities of daily living (including making a meal, using public transport, or using the telephone) and found benefits in personal activities of daily living (a secondary outcome) at the end of treatment but not at the end of scheduled follow-up. ${ }^{10}$ We are aware of more trials than were included in this review and in addition, occupational therapy is often given in settings other than the community, and its prime target is often to improve personal activities of daily living.

We conducted a systematic review to test the hypothesis that occupational therapy aimed at encouraging people to participate in personal activities of daily living after stroke will improve the recovery of ability to perform such activities.

\section{METHODS}

\section{Eligibility criteria}

We sought any randomised controlled trials that compared an occupational therapy intervention focused on activities of daily living with no routine input as the control intervention. The interventions had to be delivered by, or under the supervision of, a qualified occupational therapist. Our primary outcome of interest was independence in personal activities of daily living at the end of scheduled follow-up. The second primary outcome of interest was the extent to which participants had poor outcome, defined as death or deterioration of ability or dependency in personal activities of daily living. Secondary outcomes were death, institutionalisation, extended personal activities of daily living necessary for maintaining a dwelling in a given sociocultural setting (for example, preparing own meals, doing light housework, managing own money, shopping for personal items), patients' mood and quality of life, carers' mood and quality of life, and patients' and carers' satisfaction with services.

\section{Search strategy for the identification of studies}

We followed the search strategy developed for the stroke group of the Cochrane collaboration. ${ }^{11}$ This comprised a search of the Cochrane stroke group trials register (last searched by the review group coordinator on 7 November 2006), the Cochrane central register of controlled trials (Cochrane Library, issue 4, 2007), electronic bibliographic databases including Medline, Embase, CINAHL, PsycLIT, AMED, Wilson Social Sciences Abstracts, and the following Web of Science databases: Science Citation Index (1945 to March 2007), Social Science Citation Index (1956 to March 2007), Arts and Humanities Citation Index, dissertation abstracts register, and the occupational therapy research index. Other strategies to ensure identification of all potentially relevant trials included scanning reference lists of relevant articles and original papers, personal communication with authors, and hand searching journals. For full details of all journals searched, with dates, please see the full review in the Cochrane Library. ${ }^{12}$ One reviewer read the titles of all the references identified and eliminated any obviously irrelevant studies - for example, pharmacological or surgical interventions and study designs other than randomised controlled trials. The abstracts of the remaining studies were obtained and selected according to the assessment of two reviewers. Differences in opinion regarding trial eligibility were resolved by consensus.

\section{Data extraction}

Two reviewers independently rated the methodological quality of studies using recognised criteria ${ }^{13}$ : method of randomisation, allocation concealment, blinding of outcome assessment, and use of an intention to treat analysis. We aimed to obtain standardised data through collaboration with the original trialists. Two independent reviewers extracted data using a standard data recording form.

\section{Data analysis}

We performed an intention to treat analysis to reduce potential biases (follow-up, publication, and reporting) associated with extracting data from published reports. We obtained original trial data for eight ${ }^{\mathrm{w17} \text { w18 w20-w25 }}$ of the nine studies. This enabled a uniform approach to re-analysis of the data and standardisation of out comes.

Eight studies used individuals as the unit of randomisation and analysis ${ }^{\mathrm{w17}-\mathrm{w} 23 \mathrm{w} 25}$; one study used a randomised cluster trial design where the unit of randomisation was the nursing home. ${ }^{\text {w24 }}$ The data from the cluster randomised trial were analysed for the number of events (participants worse or dead) at the individual level using data for each participant in each cluster. We used an intracluster correlation coefficient of 0.02 to calculate the design effect and effective sample size. ${ }^{14}$

Review Manager 4.27 was used for the statistical analysis. ${ }^{15}$ Binary outcomes were analysed with a fixed effect model, as Peto odds ratios with 95\% confidence intervals. For continuous outcomes, we used the standardised mean difference with a random effects model to take account of statistical heterogeneity. 


\section{Table 1| Description of trials included in review*}

Study Sample size, characteristics, and theoretical

(setting)

Corr 195 (UK age 75.5,37\% men. Median Barthel index sco

outreach) $14(0-20)$. Clinical definition of stroke. Patients recruited before discharge from inpatient

facility. Inclusion criteria: discharged alive from one of two stroke units regardless of discharg destination. Model of human occupation

Gilbertson 138 patients: 67 intervention, 71 control. $2000^{\text {w18 }}$ (UK Median age 69, 45\% men. Median Barthel hospital index at baseline: intervention 17 (15-18), outreach) control 18 (16-19). Clinical definition of strok Patients recruited when discharged from hospital/date set. Inclusion criteria: discharged to private address; willing to cooperate; consent. Exclusion: made full recovery; discharged to institutional care; terminally ill; lived outside catchment area; severe cognitive or communication difficulties preventing consent, goal setting or completing outcome measures. Model of occupational performance

Chiu 53 patients: 30 intervention, 23 control. Mean $2004^{\mathrm{w} 19} \quad$ age $72.1,66 \%$ men. Barthel index at baseline: (Hong Kong NA. Definition of stroke: unclear. Recruitment: hospital inpatients and outpatients discharged from outreach) hospital for $<2$ weeks. Inclusion criteria: aged $>55$, diagnosis of stroke, able to follow instructions, able to communicate using speech, family support at home, required bathing device

Drummond 65 patients: 42 intervention (21 in leisure $1995^{\mathrm{w} 20}$ (UK intervention group, 21 in ADL intervention community) group), 23 control. Mean age 66, 57\% men. Barthel index at baseline: not collected. Definition of stroke: unclear. Patients recruited at discharge from inpatient facility. Inclusion criteria: admitted to hospital stroke unit. Exclusion criteria: severe comprehension difficulties (score $<3$ on Boston diagnostic aphasic examination); documented history of dementia; no English language

Walker 30 patients: 15 intervention, 15 control. Mean $1996^{\text {w21 }}$ (UK age 68,53\% men. Barthel index at baseline: not community) collected. Definition of stroke: unclear. Patients recruited at discharge from inpatient facility. Exclusion criteria: blind, deaf, unable to understand or speak English before stroke
Leisure versus conventional occupational therapy versus no occupational therapy. Leisure intervention: patients hobbies and interests were discussed in detail and the importance of maintaining a leisure programme stressed. Treatment reflected personal preferences and abilities. Help and advice included: treatment (eg practice of transfers and dressing practice needed for leisure pursuits); positioning; provision of equipment; adaptations; advice on obtaining financial assistance and transport; liaison with specialist organisations; and providing physical assistance. Conventional OT: OT activities such as transfers, washing and dressing practice, and when appropriate, perceptual treatments. Patients seen by OT for minimum of $30 \mathrm{~min} /$ week for 3 months, then $30 \mathrm{~min} /$ every 2 weeks up to 6 months; $98.5 \%$ followed

Domiciliary occupational therapy versus no occupational therapy

intervention. Domiciliary occupational therapy over a three month period provided by a senior occupational therapist. Components of intervention: dressing practice on a regular basis; teaching patients and carers specific dressing techniques, energy conservation techniques, advice on clothing adaptation. Relative/carer involvement in therapy programme and "homework" between therapy sessions. Occupational therapy provided by a qualified occupational therapist. Amount of therapy provided at therapist's discretion. Mean 6 visits over 6 months; $100 \%$ followed

Logan 111 patients: 53 intervention, 58 control. Mean $1997^{\mathrm{w} 22}$ (UK age 55, 43\% men. Barthel index at baseline:

community) NA. Clinical definition of stroke. Inclusion criteria: first stroke and discharged from hospital and referred to social services occupational therapy department

Walker 185 patients: 94 intervention, 91 control. Mean $1999^{\text {w23 }}$ (UK age 74; 51\% men. Median Barthel index at community) baseline: intervention 18 (15-20); control 18 (15-20). Clinical definition of stroke. Patients recruited $<1$ month after stroke onset from home. Exclusion criteria: >1 month after stroke onset, history of dementia, living in nursing or residential home, unable to speak or understand English before stroke

Sackley 12 nursing homes. 118 residents: 63

$2006^{\text {w24 }}$ (UK intervention, 55 control. Mean age 87.5, 19\%

community men. Mean Barthel index at baseline:

nursing

home

Enhanced occupational therapy service versus usual care. Enhanced (dedicated, prompt, and intensive) occupational therapy service provided by social services, includes provision of equipment and appliances. Occupational therapy provided by a qualified occupational therapist. Single therapist. Duration 6 months; $85.6 \%$ followed

Occupational therapy versus no occupational therapy. Occupational therapy intervention for a period of five months. Aim of therapy was to achieve independence in personal (bathing, dressing, feeding, stair mobility) and instrumental activities of daily living (outdoor mobility, driving a car, using public transport, household chores). Homework tasks were set in between therapy sessions. Occupational therapy provided by a qualified occupational therapist. Single therapist. Frequency of visits arranged between therapist, patient, and carer (if appropriate). Mean of 5.8 visits/patient over 6 months; $95.1 \%$ followed

Occupational therapy versus standard care. Occupational therapy included activities of daily living practice, mobility practice, assessment and goal setting, communication with residents, staff, relatives, and other index Definition of stroke: unclear. Inclusion criteria: $\quad 8.5$, mean total time 4.7 hours/patient over 6 months; $100 \%$ followed

Outcomes recorded at 3 and None

Outcomes recorded at 3 and None 6 months. Nottingham 3 andended index. Nottingham health profile. Nottingham leisure questionnaire.

Baseline differences

Death, Barthel index, Nottingham extended ADL index, Geriatric depression scale (short form), Pearlman's 6 point quality of life scale. Carer: Pearlman's 6 point women in intervention group $(P=0.03)$

Outcomes recorded at 7 weeks and 6 months. Primary outcomes: Nottingham extended ADL index; Favour control deterioration) in Secondary outcomes: Barthel index; Canadian occupational performance measure; EuroQol; satisfaction with outpatient services; resource use (staff time, hospital readmission, provision of equipment and services). Carer: general health questionnaire at 6 weeks

Outcomes recorded 3 months after None discharge. Primary outcome: NS. independence measure (FIM); users evaluation of satisfaction with assistive technology

Outcomes recorded at 3 and None

tingham stroke

dressing assessment. Rivermead
ADL scale. Nottingham health profile

6 months. Nottingham extended ADL index. Barthel index. General health questionnaire. Carer: general health questionnaire

Outcomes recorded at 6 months. Primary outcomes: Nottingham extended ADL index; Barthel index Barthel «15. No specific approach 

hospital age 72,71,72. Median Barthel index at

outreach) baseline: leisure 18 (15-19); ADL 18 (16-20); control $18(16-19), 58 \%$ men. WHO definition of stroke. Patients recruited from one of four participating sites at discharge. All attending stroke outcome clinic (site 5) with stroke onset< 6 months. Exclusion criteria: discharge to a nursing or residential home, recorded history of dementia, inability to complete outcome questionnaires because of limited use of English, unable to endure interventions because co-morbidity, lived outside catchment area
OT leisure $v$ ADL $v$ no OT for up to 6 months after recruitment. Leisure group: goals were set in terms of leisure activities as well as ADL tasks to achieve leisure objectives. ADL group: goals set to improve independence in self care activities and included practice in activities such as meal preparation and walking outdoors. Control group: no OT. OT provided by qualified therapist. At least 10 sessions, each at least $30 \mathrm{~min} /$ patient over 12 months; $79 \%$ followed
Outcomes recorded at 6 (primary) and 12 months. Primary outcome measure: general health questionnaire 12 item; Nottingham leisure questionnaire; Nottingham extended ADL index. Secondary outcomes: international stroke trial outcome questions; Rankin scale; Oxford handicap scale; Barthel index; London handicap scale. Carer: general health 12 item questionnaire

$\mathrm{IQR}=$ interquartile range, $\mathrm{NS}=$ not stated, $\mathrm{NA}=$ not available, $\mathrm{OT}=$ occupational therapy, $\mathrm{ADL}=$ activities of daily living.

*Unit of randomisation and analysis was individual except in w24, which was nursing home with individual adjusted for clustering.

Statistical heterogeneity between studies was examined with $\chi^{2}$ and $I^{2} .{ }^{16}$ An $I^{2}$ value over $50 \%$ was considered to indicate substantial inconsistency. Publication bias was assessed with a rank correlation test and a funnel plot. ${ }^{17}$

We planned sensitivity analyses to explore the influence of the method of randomisation, allocation concealment, blinding of final outcome assessment, and the presence of an intention to treat analysis.

\section{RESULTS}

Figure 1 outlines the results of the trial selection process. We identified 14593 references from the searches, of which 14528 were excluded from title or abstract, leaving 65 potentially eligible studies for inclusion. After we obtained full texts for these studies, we then excluded 54 as they did not fulfil the inclusion criteria. Reasons for exclusion were as follows: intervention provided by a healthcare professional other than occupational therapist (17 studies), multidisciplinary intervention including occupational therapy (eight), intervention not focused on personal activities of daily living (15), one type of occupational therapy versus another type of occupational therapy (six), ${ }^{\text {wl-w6 }}$ not a randomised controlled trial (five), ${ }^{\text {w7-w11 }}$ and insufficient numbers of stroke participants (three), ${ }^{\text {w12-w14 }}$ (detailed exclusions are given in the Cochrane Library version of the review ${ }^{12}$ ). Two trials are not yet completed. $^{\mathrm{w} 15 \mathrm{w} 16}$ The remaining nine studies were included in the review and contained information on 1258 participants. $^{\text {w17-w25 }}$ Table 1 gives details of the included studies. Table 2 provides information on the methodological quality of the included studies, and table 3 describes the six trials that we excluded from the review because they did not have a suitable control group.

The mean age of participants in studies ranged from 55 to 87.5 years and the proportion of men ranged from $19 \%$ to $66 \%$. Baseline scores on the Barthel index ${ }^{18}$ were available for five trials. ${ }^{\text {w17 w18 w23-w25 Four }}$ trials included people with mild to moderate disability

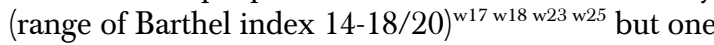
trial recruited more severely dependent participants (mean Barthel index 9-10/20). ${ }^{\text {224 }}$ Exclusion criteria were communication difficulties and cognitive or other co-existing conditions that would interfere with compliance or outcome assessment ${ }^{\mathrm{w} 18-\mathrm{w} 21 \mathrm{w} 23 \mathrm{w} 25}$; inability to speak English $^{\text {w20 w21 w23 w25 }}$; terminal illness ${ }^{\text {w18 }}{ }^{\text {w19 }}$; residence in, or about to be discharged to, a residential or nursing home ${ }^{\text {w18 w23 w25 }}$; not living at home and without carer or family support ${ }^{\mathrm{w} 19}$; and a Barthel score over 15. ${ }^{\text {w24 }}$ One trial recruited participants who had not been admitted to hospital after stroke onset, ${ }^{\text {w23 }}$ and another trial recruited only from nursing homes. ${ }^{\text {w24 }}$ Six trials recruited from inpatient facilities. ${ }^{\text {w17w18w20-w22 w25 }}$ One trial recruited participants two weeks after discharge from inpatient facilities. ${ }^{\text {w19 }}$

Most studies had parallel groups with occupational therapy focused on personal activities of daily living compared with usual care or no routine intervention. Two trials compared two alternative interventions (occupational therapy based on leisure activities or personal activities of daily living) against usual care or no routine intervention in three parallel groups. One trial used a crossover design in which participants were given dressing practice followed by the personal activities of daily living intervention of interest, in sequence. ${ }^{\text {w21 }}$ For further details of the interventions provided, see the Cochrane review. ${ }^{12}$

Eight trials clearly described concealed allocation, randomisation procedures, an objective, and explicit blinded outcome assessment for all participants. $^{\text {w17 w18 w20-w25 }}$ Four studies explicitly reported the use of an intention to treat

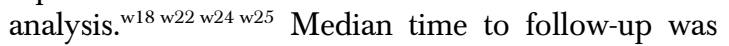
six months (range 3-12 months). Rates of loss to follow-up varied considerably across the reported outcomes. Sixty one (8.5\%) participants from the intervention groups and $34(6.3 \%)$ from the control groups died during follow-up.

\section{Personal activities of daily living}

Six studies used the Barthel index ${ }^{18}$ to measure personal activities of daily living, ${ }^{\text {w17 w18 w22-w25 }}$ one study used the self care section of the Rivermead personal activities of daily living scale, ${ }^{19}$ w21 and one study used the functional independence measure. ${ }^{20}$ w19 $\mathrm{A}$ score for personal activities of daily living was available for 
Table 2 | Quality assessment of trials included in review

\begin{tabular}{|c|c|c|c|c|c|}
\hline Study & $\begin{array}{c}\text { Appropriate } \\
\text { randomisation and } \\
\text { allocation concealment }\end{array}$ & $\begin{array}{l}\text { Unbiased data } \\
\text { collection }\end{array}$ & $\begin{array}{l}\text { Follow-up } \\
\geq 95 \%\end{array}$ & $\begin{array}{l}\text { Length (months) and success of } \\
\text { follow-up on primary outcome }\end{array}$ & $\begin{array}{l}\text { Difference in attrition } \\
\text { between groups } \leq 5 \%\end{array}$ \\
\hline Corr $1995^{\mathrm{w} 17}$ & Yes & Yes & Yes & $12 ; 95.5 \%$ & No $(9 \%)$ \\
\hline Gilbertson $2000^{\text {w18 }}$ & Yes & Yes & Yes & $6 ; 96.4 \%$ & Yes (5\%) \\
\hline Chiu $2004^{\text {w19 }}$ & No & No & Yes & $3 ; 100 \%$ & Yes $(0)$ \\
\hline Drummond $1995^{\mathrm{w} 20}$ & Yes & Yes & Yes & $6 ; 100 \%$ & Yes (0) \\
\hline Walker $1996^{\text {w21 }}$ & Yes & Yes & No & $6 ; 90 \%$ & No $(20 \%)$ \\
\hline Logan $1997^{\text {w22 }}$ & Yes & Yes & No & $6 ; 85.6 \%$ & No $(16 \%)$ \\
\hline Walker $1999^{\text {w23 }}$ & Yes & Yes & Yes & $6 ; 95.1 \%$ & No $(1 \%)$ \\
\hline Sackley $2006^{\text {w24 }}$ & Yes & Yes & Yes & $6 ; 100 \%$ & Yes (0\%) \\
\hline Parker $2001^{\text {w25 }}$ & Yes & Yes & No & $12 ; 79 \%$ & Yes (3\%) \\
\hline
\end{tabular}

$961(80.6 \%)$ participants from eight trials. ${ }^{\text {w17-w19 w21-w25 }}$ The pooled result for all trials, combined as a standardised mean difference, was 0.18 (95\% confidence interval 0.04 to $0.32 ; \mathrm{P}=0.01$ ) with no significant heterogeneity $(\mathrm{P}=0.33)$ (fig 2). Therefore, participants who received occupational therapy after stroke were significantly more independent in personal activities of daily living than those who received no intervention or usual care. The estimated standardised mean difference of 0.18 is equivalent to a one point $(5 \%)$ difference on the 20 point Barthel index, assuming a population $\mathrm{SD}$ of six points.

There was no substantial change in results when we limited sensitivity analyses to the seven trials with clear allocation, randomisation procedures, or

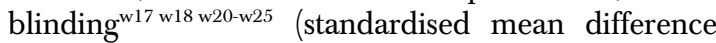
$0.17,0.02$ to $0.33 ; \mathrm{P}=0.03)$. When we restricted analysis to the four trials that performed an intention to treat

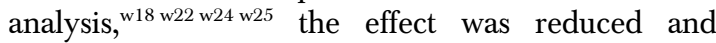
became non-significant $(0.12,0.10$ to $0.33 ; \mathrm{P}=0.28)$.

In our post hoc analysis excluding the leisure based occupational therapy arms from the two trials $\mathrm{s}^{\mathrm{w} 20 \mathrm{w} 25}$ that compared alternative forms of intervention (occupational therapy based on leisure activities or personal activities of daily living), we found similar results $(0.20$, 0.06 to $0.33 ; \mathrm{P}=0.004)$ with no significant heterogeneity $(\mathrm{P}=0.56)$.

\section{Deterioration in personal activities of daily living}

The second outcome concerned the extent to which occupational therapy could influence the risk of deterioration in personal activities of daily living. We defined this as the combined "poor outcome" of death or experiencing a deterioration in ability to perform personal activities of daily living (experiencing a drop of one or more points in a given score for personal activities of daily living) or dependent (below a predefined threshold on a given personal activities of daily living scale; for the Barthel index this was 15), or requiring institutional care at the end of scheduled follow-up. Data on poor outcome were available for 1065 $(90.6 \%)$ participants from seven trials ${ }^{\mathrm{w} 17}$ w18 w20 w22-w25 and showed that the odds of a poor outcome were significantly lower in the participants who received occupational therapy (odds ratio 0.67, 0.51 to 0.87; $\mathrm{P}=0.003$ ) with no significant heterogeneity between studies $(\mathrm{P}=0.28)$ (fig 3$)$. The overall rate of a poor outcome for controls was $42 \%$, which combined with an odds ratio of 0.67 gives an estimated number needed to treat of 11 (7 to 30).

Re-analysis for the outcome death and deterioration in the score for personal activities of daily living included information on 407 (98.5\%) participants from four trials ${ }^{\mathrm{w} 17 \mathrm{w} 18 \mathrm{w} 20 \mathrm{w} 24}$ and produced similar results (odds ratio $0.60,0.39$ to $0.91 ; \mathrm{P}=0.02$ ) with no significant heterogeneity. Further analysis with exclusion of the leisure based occupational therapy arms from the two trials ${ }^{\mathrm{w} 20 \mathrm{w} 25}$ that compared alternative forms of interventions (occupational therapy based on leisure or personal activities of daily living) provided similar results (odds ratio $0.65,0.49$ to 0.86 ; $\mathrm{P}=0.002)$ with no significant heterogeneity between studies $(\mathrm{P}=0.37)$.

There was no substantial change in results when we conducted sensitivity analyses excluding trials with clear intention to treat analysis. If we assume that the participants who were missing $(66 / 673(9.8 \%)$ in intervention groups and 44/502 (8.8\%) in control groups) had a poor outcome, then the odds of a poor outcome remained significantly reduced for those participants who received occupational therapy (odds ratio 0.67 , 0.52 to $0.86 ; \mathrm{P}=0.002)$ with no significant heterogeneity $(\mathrm{P}=0.27)$. Furthermore, if we assume that the participants who were missing from the treatment groups were alive and well and living at home, then the odds of a poor outcome were still significantly reduced for those who received occupational therapy (odds ratio $0.71,0.55$ to $0.92 ; \mathrm{P}=0.009$ ) with no significant heterogeneity $(\mathrm{P}=0.20)$.

We found no evidence of publication bias from the rank correlation test for the outcome death or "poor outcome" $(\mathrm{P}=0.108$, seven studies) or in the funnel plot.

\section{Secondary outcomes}

We had scores on the Nottingham extended activities of daily living scale for $847(78.8 \%)$ participants from

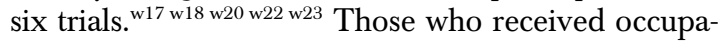
tional therapy were significantly more independent in instrumental activities of daily living (standardised mean difference $0.21,0.03$ to $0.39 ; \mathrm{P}=0.02$ ). There was a non-significant benefit in mood or distress scores 
Table 3 | Description of six trials of occupational therapy for stroke excluded from review

\section{Study (setting)}

Donkervoort 2001 ${ }^{\mathrm{w1}}$

(inpatients)
Participants

113 participants, 56 strategy training, 57 usual occupational therapy. Mean age $65.4 ; 52 \%$ men. Inclusion criteria: left hemisphere stroke, criteria: history of apraxia before current stroke, index stroke onset 44 weeks, aged 225 or $>95$, history of post-traumatic brain damage, history of brain tumour, unable to speak Dutch, premorbid or current psychiatric, psychogeriatric, addiction to alcohol or other drugs, premorbid personality, intellectual or learning disorder, history of severe consciousness impairments. Assessed not to require treatment

Edmans $2000^{\mathrm{w} 2}$ (inpatients) 80 participants, 40 in transfer of training group and 40 in functional training group. Inclusion criteria: sufficient cognitive, language, and functional ability to complete the Rivermead perceptual assessment battery, sufficient functional use of one hand to complete perceptual treatment activities, consent
Jongbloed $1989^{\text {w3 }}$ (inpatients)

90 participants, 43 in sensorimotor integrative treatment group and 47 in functional treatment group. Mean age $71.32 ; 45 \%$ men. Inclusion criteria: admitted to hospital $\leq 12$ weeks after first CVA, presented with unilateral upper and lower extremity weakness on admission to hospital, no experience of nursing, residential, or extended care before admission to hospital, no severe aphasia, able to consent

Lui $2001^{\text {w4 }}$ (inpatients) 22 participants, 12 intervention and 10 control. Mean age $71.3 ; 54 \%$ men. Inclusion criteria: unilateral stroke, independent in activities of daily living before stroke, able to communicate, medically stable

Morgan $2002^{\mathrm{w} 5}$ (hospital Inclusion criteria: men $>40$ and $<50$ years, first outreach) stroke, middle cerebral artery syndrome of thromboembolic origin confirmed by CT, middle band in Garraway and coworkers neurological screening process. Exclusion criteria: considerable complications or comorbidities after stroke, any impairment that would prevent use of Canadian occupational performance measure such as aphasia

Young $1983^{\text {w6 }}$ (unclear)

\section{7 participants (9 per group). Mean age 64.15.} Inclusion criteria: right CVA, age 45-80, assessed to have left neglect or visual deficits, or both. Exclusion: history of alcoholism, psychiatric treatment, or previous neurological impairment
Transfer of training approach $v$ functional approach to treatment perceptual problems. Perceptual treatment given for 2.5 hours/week for 6 weeks. Rivermead perceptual assessment battery, Barthel activities of daily living index, and Edmans activities of daily living index

Sensorimotor integrative treatment Compared two types of techniques $v$ functional treatment 40 occupational therapy $\mathrm{min} /$ day, 5 days/week for 8 weeks.

Barthel index, meal preparation, sensorimotor integration tests

Connectionist model (task generalisation programme) $v$ traditional learn task strategy on daily

tasks. Evaluated on performance of tasks

Client centred occupational therapy intervention programme $v$ therapist led functional occupational therapy programme. Modified motor assessment scale, modified Barthel index, Canadian occupational performance measure Hour of routine occupational therapy/day $v 20$ min routine Compared different intensities occupational therapy $+20 \mathrm{~min}$ of occupational therapy

cancellation training +20 min visual scanning training $v 20$ min block design training +20 min cancellation training +20 min of visual scanning training. Letter cancellation task, wide range achievement test, copying and address, counting faces, activities of daily living (outcome measure not stated) Compared two types of

Compared two types of occupational therapy occupational therapy

Compared two types of occupational therapy

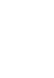




\begin{tabular}{|c|c|c|c|c|c|c|c|c|}
\hline \multirow{3}{*}{$\begin{array}{l}\text { Study } \\
\text { Corr } 1995^{\mathrm{w} 17}\end{array}$} & \multicolumn{2}{|c|}{ Treatment } & \multicolumn{2}{|r|}{ Control } & & \multirow{2}{*}{\multicolumn{2}{|c|}{$\begin{array}{l}\text { Standard mean difference } \\
\quad(\text { random })(95 \% \mathrm{Cl})\end{array}$}} & \multirow{2}{*}{$\begin{array}{l}\text { Standard mean differenc } \\
\text { (random) }(95 \% \mathrm{Cl})\end{array}$} \\
\hline & No & Mean (SD) & No & Mean (SD) & & & & \\
\hline & 46 & $12.30(4.74)$ & 39 & $10.87(5.72)$ & & - & & $0.27(-0.16$ to 0.70$)$ \\
\hline Gilbertson $2000^{\mathrm{w} 18}$ & 60 & $16.17(3.76)$ & 62 & $15.45(4.48)$ & & & & 0.17 (-0.18 to 0.53$)$ \\
\hline Chiu $2004^{\mathrm{w19}}$ & 30 & $108.90(11.60)$ & 23 & $104.90(12.00)$ & & & & $0.33(-0.21$ to 0.88$)$ \\
\hline Walker $1996^{\text {w21 }}$ & 12 & $10.75(3.86)$ & 15 & $10.33(4.19)$ & & & & $0.10(-0.66$ to 0.86$)$ \\
\hline Logan $1997^{\mathrm{w} 22}$ & 45 & $15.42(4.64)$ & 38 & $14.82(3.97)$ & & & & $0.14(-0.30$ to 0.57$)$ \\
\hline Walker $1999^{\text {w23 }}$ & 84 & $18.44(2.72)$ & 79 & $17.35(3.05)$ & & & - & $0.38(0.07$ to 0.69$)$ \\
\hline Sackley $2006^{\text {w24 }}$ & 53 & $10.21(5.90)$ & 47 & $8.09(4.45)$ & & & & 0.40 (0.00 to 0.80$)$ \\
\hline Parker $2001^{\text {w25 }}$ & 218 & $15.77(4.04)$ & 110 & $16.08(3.87)$ & & $\longrightarrow$ & - & $-0.08(-0.31$ to 0.15$)$ \\
\hline Total (95\% Cl) & 548 & & 413 & & & & & 0.18 (0.04 to 0.32 ) \\
\hline \multicolumn{5}{|c|}{ Test for heterogeneity: $\chi^{2}=8.08, \mathrm{df}=7, \mathrm{P}=0.33, I^{2}=13.3 \%$} & -1 & -0.5 & 0.5 & 1 \\
\hline \multicolumn{5}{|c|}{ Test for overall effect: $z=2.45, P=0.01$} & $\begin{array}{l}\text { Favours } \\
\text { control }\end{array}$ & \multicolumn{3}{|c|}{$\begin{array}{r}\text { Favours } \\
\text { treatment }\end{array}$} \\
\hline
\end{tabular}

\section{Fig 2 | Effects of occupational therapy on personal activities of daily living}

Thirdly, it is more difficult to obtain acceptance of randomisation in an inpatient setting, particularly where an occupational therapy service is already established. We excluded four trials that compared one occupational therapy intervention within an active concurrent control arm provided in inpatient settings as they did not provide an unconfounded estimate of effect. ${ }^{\text {wl-w4 }}$ Finally, trials of rehabilitation interventions typically have lengthy follow-up periods with a risk of study dropout. This makes performing a true intention to treat analysis with complex scores such as the Barthel index problematic as it is difficult to score for missing participants. Despite these potential concerns, however, the quality of the included trials was generally good and the results were consistent between trials.

Occupational therapy is a complex intervention. Practice includes skilled observation; the use of standardised and non-standardised assessments of the biological, psychiatric, social, and environmental determinants of health; clarification of the problem; formulation of individualised treatment goals; and the delivery of a set of individualised problem solving interventions. While we are confident that all the interventions in this review were consistent with this broad

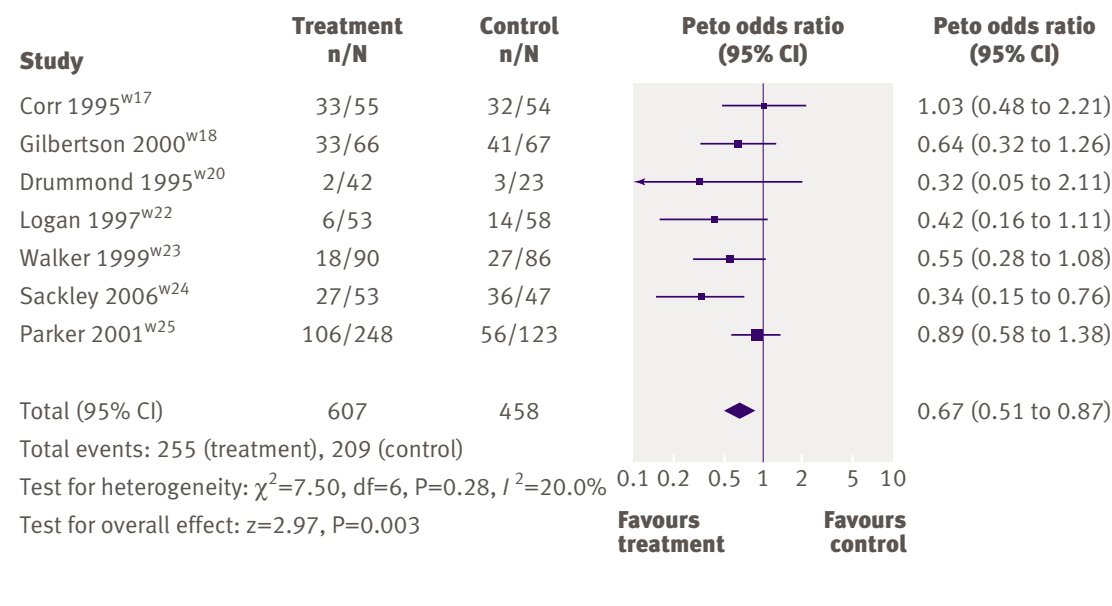

Fig 3 | Effects of occupational therapy on poor outcome concept of occupational therapy, we recognise that the exact nature of the interventions in each study differed according to the type of patient, the expertise of the therapist, and the resources available. The interventions tested were probably provided by experts and not particularly constrained by day to day service factors. Our review did not compare occupational therapy with alternative rehabilitation interventions, nor did it examine the effect of occupational therapy combined with other interventions.

\section{Comparison with previous studies}

Previous reviews that have assessed the role of occupational therapy either have not specifically focused on stroke, ${ }^{22}$ have concentrated on instrumental activities of daily living in the subgroup of stroke patients living in the community, ${ }^{10}$ or have included a wide range of studies of varying methodological quality. ${ }^{23}$ Our review adds substantially to the literature by examining the effects of occupational therapy focused personal activities of daily living in stroke patients regardless of treatment setting.

\section{Implications for research}

Occupational therapy after stroke "works" in that it improves outcome in terms of ability in personal activities of daily living. The estimate that 11 (7 to 30) patients need to be treated to avoid one patient deteriorating in personal activities of daily living should be regarded as an approximate indicator. This is a relatively crude measure of outcome, which does not capture potential benefits in other domains of health. This figure also suggests, however, that not all patients treated by an occupational therapist will benefit. Further work is required to define those individuals who are most likely to benefit from occupational therapy, and economic studies are required to examine the cost effectiveness of occupational therapy. We believe that our findings should move the research agenda away from the questions surrounding whether occupational therapy (as a package of interventions) is effective to the identification of which specific interventions are effective for particular patients. 


\section{WHAT IS ALREADY KNOWN ON THIS TOPIC}

Reviews of rehabilitation therapies show that they improve personal abilities in activities of daily living in people who have had a stroke, but the individual contribution from occupational therapy is not certain

Previous reviews of trials of occupational therapy in stroke have not specifically studied such personal ability

\section{WHAT THIS STUDY ADDS}

Occupational therapy is an effective intervention to improve personal ability in activities of daily living in patients who have had a stroke

Around 11 (95\% confidence interval 7 to 30 ) people with stroke would need to be treated to avoid a poor outcome in one person
7 De Wit L, Putman K, Lincoln N, Baert I, Berman P, Beyens H, et al. What do physiotherapists and occupational therapists actually do? Stroke 2006;37:1483-9.

8 Stroke Unit Trialists' Collaboration. Organised inpatient (stroke unit) care for stroke (Cochrane Review). Cochrane Database Syst Rev 2001;(3):CD000197.

9 Legg L, Langhorne P, Outpatient Service Trialists. Rehabilitation therapy services for stroke patients living at home: systematic review of randomised trials. Lancet 2004;363:352-6.

10 Walker MF, Leonardi-Bee J, Bath P, Langhorne P, Dewey M, Corr S, et al. Individual patient data meta-analysis of randomized controlled trials of community occupational therapy for stroke patients. Stroke 2004;35:2226-32.

11 Sandercock P, Anderson C, Bath P, Bereczki D, Candelide L, Chen C, et al. Cochrane stroke group. In: Cochrane Library, Issue 4. Oxford: Update Software, 2002.

12 Legg LA, Drummond AE, Langhorne P. Occupational therapy for patients with problems in activities of daily living after stroke. Cochrane Database Syst Rev 2006;(4):CD003585.

This study was done as a Cochrane systematic review under the auspices of the Cochrane Stroke Group, whose invaluable assistance is gratefully acknowledged.

Contributors: $L L$ and $A D$ planned the review. $L L$ was lead reviewer and produced the first draft of the paper. AD, PLa, JL-B, and JRFG all collaborated on the final version before initial submission and took responsibility for the submitted version of the paper. SC, MD, JE, LG, LJ, PLo, CS, and MW were members of the occupational therapy trialists and obtained primary data and assisted in the editing of the paper. LL is guarantor.

Funding: The Big Lottery Fund and Chest Heart and Stroke Scotland funded staff time.

Competing interests: None declared.

Ethical approval: Not required.

Provenance and peer review: Not commissioned; externally peer reviewed.

1 Murray CJL, Lopez AD. Mortality by cause for eight regions of the world: global burden of disease study. Lancet 1997;349:1269-76

2 Wade DT, Langton-Hewer R. Functional abilities after stroke: measurement, natural history and prognosis. J Neurol Neurosurg Psychiatry 1987;50:177-82.

3 Hopson S. The principles of activities of daily living. In: Turner A, ed. The practice of occupational therapy. An introduction to the treatment of physical dysfunction. London: Church-Livingston, 1981:31.

4 Sulter G, Steen C, De Keyser J. Use of Barthel index and modified Rankin scale in acute stroke trials. Stroke 1999;30:1538-41.

5 Langhorne $P$, Pollock $A$. What are the components of effective stroke unit care? Age Ageing 2002;31:365-71.

6 American Occupational Therapy Association. Policy 5.3.1: Definition of occupational therapy practice for state regulation. Am J Occup The 1994;48:1072-3. 